\title{
VIVENCIAS DE RECONOCIMIENTO Y COMPASIÓN DE PERSONAS EN CONDICIÓN DE DISCAPACIDAD. HACIA LA AUTONOMÍA Y LA VIDA INDEPENDIENTE ${ }^{1}$
}

\section{Recognition and compassion experiences of people with disabilities. Towards autonomy and independent living}

\author{
Natali RoldÁn BERRIo \\ Universidad Católica Luis Amigó, Medellín, Colombia \\ natiroldanb@yahoo.com \\ Magda Victoria Díaz Alzate \\ Universidad Católica Luis Amigó, Medellín, Colombia \\ magda.diazal@amigo.edu.co
}

Recepción: 6 de julio de 2018

Aceptación definitiva: 6 de febrero de 2019

Resumen: La condición de discapacidad ha sido percibida como restrictiva para el desarrollo de la autonomía y la vida independiente, lo que profundiza los sentimientos de limitación de las personas en esta condición. Este artículo presenta los principales resultados de una investigación cualitativa realizada con cuatro participantes que se propuso comprender vivencias de reconocimiento y compasión de participación de personas en condición de discapacidad, que favorecen la autonomía y la configuración de estilos de vida independientes, para trascender la percepción negativa de esta condición. Se encontró que estas vivencias, favorecieron el desarrollo de la confianza en uno mismo y la capacidad para reconocer la importancia de los apoyos para una vida digna, además, que las personas en esta condición, cuando tienen experiencias de reconocimiento y compasión, logran entender que la autonomía es un objetivo que se alcanza para vivir

1 Artículo derivado de la investigación "Vivencias de reconocimiento y compasión de personas en condición de discapacidad: transitar la autonomía hacia un estilo de vida independiente", realizada entre julio de 2016 y julio de 2018. Maestría en Intervenciones Psicosociales. Grupo de Investigación Estudios de Fenómenos Psicosociales, Línea Problemáticas Psicosociales Contemporáneas. Universidad Católica Luis Amigó. Medellín, Colombia. 
la cotidianidad con los apoyos necesarios y que la independencia se refiere a la capacidad de tomar decisiones sobre la propia vida.

Palabras Clave: condición de discapacidad; reconocimiento; compasión; autonomía; vida independiente.

Aвstract: The condition of disability has been perceived as restrictive for the development of autonomy and independent life, which deepens the feelings of limitation of people in this condition. This article presents the main results of a qualitative research carried out with four participants that aimed to understand experiences of recognition and compassion of the participation of people in a disability condition, that favor the autonomy and the configuration of independent lifestyles, to transcend the negative perception of this condition. It was found that these experiences favored the development of self-confidence and the ability to recognize the importance of support for a dignified life, in addition, that people in this condition, when they have experiences of recognition and compassion, manage to understand that the autonomy is an objective that is achieved to live the daily life with the necessary supports, and that independence refers to the ability to make decisions about one's life.

KEY WORDS: condition of disability; recognition; compassion; autonomy; independent life.

\section{Introducción}

L

A REVISIÓN DOCUMENTAL SOBRE EL TEMA permitió identificar que no existen estudios que relacionen de manera holística las categorías de reconocimiento y compasión con estilos de vida independiente y autonomía de personas en condición de discapacidad. Se presentan estudios que tratan la importancia de reconocer la diversidad, de construir relaciones dignas con las personas en condición de discapacidad y se enfocan en las perspectivas crítica y política de la discapacidad. Las publicaciones de Pascual-García, Garrido-Fernández y Antequera-Jurado, 2014; Rojas, 2006; Baña Castro, Losada Puente y Muñoz Cantero, 2015 Pérez-Bustos, Moya-Solar y Jiménez Figueroa, 2013, tienen como punto de encuentro otorgar importancia a las vivencias de participación en diferentes escenarios como factor que incide en el desarrollo de la autodeterminación. Asimismo, se encuentran estudios sobre procesos de inclusión (Bossaert, Colpin, Pijl y Petry, 2013; Gonçalves y Lemos, 2014; Castillo y Garro-Gil, 2015; Dias, 2015; Rachmawati, Nu'man, Widiasmara y Wibisono, 2016; Arduini y Chiusaroli, 2013; Cramerotti y Ianes, 2016; Ab.Latiff, Wan y Azrani, 2015) y de participación (Allan, Smith, Côté, Martin Ginis y Latimer-Cheung, 2017), que se han concentrado en develar las condiciones institucionales educativas y familiares para favorecer $\mathrm{u}$ obstaculizar estos dos procesos. Las preguntas están en los factores ambientales y actitudinales, menos en las relaciones vinculantes o afectivas que se tejen en estos espacios, que es una perspectiva del modelo social de la discapacidad. 
Los investigadores Mattevi, Bredemeier, Fam y Fleck (2012) realizaron un estudio cuyo objetivo era explorar las percepciones sobre calidad de vida que tenían 23 personas participantes. Concluyeron que esta población puede tener concepciones únicas sobre la calidad de vida; ello es importante porque favorece el reconocimiento de sus particularidades. Por ejemplo, hallan en el ocio un factor importante para hablar de calidad de vida, lo cual también es encontrado en el estudio de Dollar, Fredrick, Alberto y Lucke (2011), quienes argumentan que el desarrollo de habilidades para la vida y del ocio permite que las personas en condición de discapacidad puedan tener una mejor calidad de vida, puesto que aumenta la autonomía y autosuficiencia.

También se encuentran desarrollos sobre la autonomía e independencia (Löve, Traustadóttir y Rice, 2017) desde enfoques críticos de las teorías sociales, que proponen que no es suficiente tener legislación, sino que se debe garantizar la vivencia de estos dos aspectos en los diferentes ámbitos de participación. Trascienden la mirada jurídica para ubicarse en la perspectiva política de los derechos de estas personas. En esta misma línea política, se encontró una revisión documental realizada por PeñaOchoa y Durán-Palacio (2015), en la cual dan cuenta de 58 textos que relacionaban el concepto de justicia con desempeño laboral, psicología del trabajo y las organizaciones. En este texto se destaca una conclusión sobre las emociones negativas hacia las personas en condición de discapacidad, como el principal obstáculo laboral, por encima de las limitaciones físicas, económicas o legales.

El estudio más cercano encontrado fue el desarrollado por Fernández y Vasco (2013) quienes, desde la perspectiva ética del reconocimiento de Honneth, analizan relatos sobre las luchas por el reconocimiento de 25 jóvenes en condición de discapacidad. Concluyeron que los jóvenes reivindican su condición y reportan vivencias de reconocimiento en algunos espacios; sin embargo, las experiencias de exclusión y marginación en las instituciones educativas acrecientan el riesgo de sufrir menosprecio.

Los vacíos hallados en la literatura existente para la comprensión de relaciones complejas que favorezcan los desarrollos de autonomía y estilos de vida independientes de las personas en condición de discapacidad, que trasciendan el modelo social preponderante en la región latinoamericana, permitieron construir la problematización con perspectiva política de la investigación que se presenta en este artículo.

Para contextualizar, es imperativo nombrar que la discapacidad, como concepto, ha transitado desde el modelo de la prescindencia, el modelo médico, el modelo social, hasta el modelo actual de la diversidad funcional (Palacios y Romañach, 2006), desarrollado en España. Los movimientos sociales que abogan por la justicia social, la equidad y la igualdad de derechos en el mundo favorecen decisiones de los gobiernos y otras organizaciones para que se reconozcan las diversidades, no solo raciales o de género, sino de condiciones como la discapacidad.

El surgimiento de iniciativas como el Movimiento de Vida Independiente (Maraña, 2004; Iáñez-Domínguez, 2009), que nace de la decisión de un grupo de personas en condición de discapacidad del mundo de reclamar los derechos políticos de decidir, elegir y construir un proyecto de vida propio, teniendo en cuenta sus limitaciones, y la Clasificación Internacional del Funcionamiento, de la Discapacidad y de la Salud -CIF- (Organización Mundial de la Salud, 2001) favorecen la comprensión 
de perspectivas políticas sobre las personas, reconociendo su condición de limitación, pero otorgando el estatus político de ciudadanos de derechos. Estos avances ponen al descubierto que la condición de discapacidad en la historia se ha entendido como padecimiento, sufrimiento y angustia, a partir de la perspectiva del modelo médicorehabilitador. En el caso de Colombia, hay un tránsito importante y desde finales de los años ochenta aumentaron las investigaciones respecto al tema (Rojas y Haya, 2017), pero las valoraciones de la discapacidad como una anormalidad que debe ser superada perpetúan las barreras para la participación de estas personas en los diferentes escenarios; además, limitan el autorreconocimiento de cuerpos diversos que merecen los derechos ciudadanos de todos los habitantes del país, puesto que ello influye en la configuración como seres sociales y políticos. La presencia de actitudes de rechazo, limitación en el acceso a la educación y a oportunidades laborales (Santamaría, Angarita, Bayona, Delgado, Parra y Pérez, 2013) afectan la valoración social, el acceso a los derechos y el surgimiento de condiciones de autonomía y formas de vida independiente.

La conceptualización de la discapacidad como condición se encuentra en un momento coyuntural por la emergencia de enfoques teóricos; las nuevas formas de comprender las vivencias de estas personas; y las diferentes relaciones que han establecido con el contexto social, político y familiar, que favorecen avances significativos para su reconocimiento como personas; además, logran que se cuestionen las constantes actitudes negativas y discriminatorias que prevalecen en la sociedad. Se parte de la idea de que los seres humanos, para vivir en armonía con su historia de vida, con la de los demás y con las condiciones que le presenta el entorno, deben hallar las herramientas y condiciones necesarias para ello. En este sentido, se requieren ámbitos que favorezcan la participación, y los reconozcan como actores sociales con necesidades, potencialidades e intereses y con formas particulares de habitar el mundo (Acosta Valencia y Garcés Montoya, 2010), para que encuentren, a través de las distintas vivencias forjadas con los otros, la reivindicación de su proyecto personal, social, familiar y laboral, y darle sentido a lo que perciben como su estilo de vivir.

Sobre el concepto de reconocimiento, se halla en la propuesta de Honneth (1992, 1996, 2006, 2009) una aproximación pertinente a la experiencia descrita por los participantes en los diferentes ámbitos de participación. Desde esta perspectiva, el reconocimiento transita por tres esferas, que no necesariamente son consecutivas, pero que sí favorecen el fin último de reconocer al otro como igual en derechos y en su dignidad, pero diverso en su condición de ser humano. Otorgar al otro un estatus moral implica que pueda tomar decisiones sobre sus proyectos de vida, lo que favorece la vida independiente, concepto que es definido por Maraña (2004) como un modelo que precisa el derecho de las personas en condición de discapacidad a tomar decisiones como ciudadano, con libertad e independencia, lo que les permitirá acceder a los mismos derechos del resto de la humanidad. Se concibe la configuración de la independencia como un derecho. Al decir de Iáñez-Domínguez (2009) “todas las personas, indistintamente cuales sean sus limitaciones y/o capacidades, tienen el derecho a demandar y obtener su independencia a través del máximo control sobre sus vidas” (p. 13). 
Para entender la autonomía, se trasciende el concepto descrito por la Comisión Económica para América Latina y el Caribe (CEPAL) (2012), que la define como "la capacidad de desempeñar las funciones relacionadas con la vida diaria, es decir, vivir en la comunidad recibiendo poca o ninguna ayuda de los demás” (p. 43), y se acude a la autonomía moral (Iáñez-Domínguez, 2009) que implica poder recibir ayuda para realizar funciones cotidianas, sin que ello necesariamente limite la toma de decisiones de las personas en condición de discapacidad sobre maneras de hacerlo, incluso, frente a determinar de quién desean recibir la ayuda. Se determina así la diferencia entre autonomía funcional que presenta la CEPAL, que necesariamente puede contener una barrera para el desarrollo de la autonomía en personas con mayores grados de limitación, en tanto exige la mínima o nula utilización de ayuda para que se defina como persona autónoma, y la autonomía moral considerada por el Movimiento de Vida Independiente como la posibilidad de entender un desarrollo más acorde a las condiciones reales de limitaciones.

Asimismo, para comprender la vivencia de la compasión, se acudió a los desarrollos teóricos de Nussbaum (2008). La compasión como una emoción que permite volcarse a la atención del otro en su humanidad pasa por tres juicios valorativos del sufrimiento que ese otro está padeciendo: la magnitud del padecimiento, el inmerecimiento del mismo y el reconocimiento de la fragilidad como condición que pone a todos en el mismo riesgo de vivir este sufrimiento, a este último Nussbaum (2008) lo nombra como eudaimonista.

Lo anterior sirvió como pretexto para la generación de la investigación de la cual se deriva este artículo y que tuvo como objetivo comprender las vivencias de reconocimiento y compasión en diferentes ámbitos de participación de personas en condición de discapacidad, que favorecen la autonomía y la configuración de un estilo de vida independiente. En este sentido, procuró la visibilización de las diversidades corporales y la aceptación del requerimiento de los apoyos y concibió a los participantes como personas activas con capacidades para apropiarse de las experiencias, y actuar en consecuencia con sus recursos personales, sus barreras y las construcciones vinculares, que aportan a su configuración subjetiva. La persona es comprendida desde una perspectiva de derechos humanos materializados en capacidades, que al decir de Nussbaum (2012) "no son simplemente objetivos sociales deseables, sino títulos basados en la justicia para una reclamación urgente” (p. 288), y que proveen, particularmente, a las personas con discapacidad para la construcción de estilos de vida independientes.

Este artículo presenta, primero, los aspectos metodológicos que evidencian la rigurosidad del estudio y que describen el procedimiento que permitió los hallazgos. Luego se da cuenta de dos categorías emergentes que fueron resultado del proceso analítico enunciado en el método, a saber: Vivencias de reconocimiento y compasión en ámbitos de socialización y participación, y Experiencias de reconocimiento y compasión, que favorecen la autonomía y configuran estilos de vida independiente. Finalmente, se presentan algunas consideraciones finales o conclusiones del estudio. 


\section{Método}

\subsection{Diseño de la investigación}

La investigación, por su carácter cualitativo, favoreció la emergencia de aspectos relevantes en el proceso. Inicialmente, se pretendía comprender las experiencias de reconocimiento y compasión vividas en las familias; sin embargo, en el proceso de entrevistas a los cuatro participantes, se encontró que ellos tenían estas vivencias en espacios más amplios de participación, lo cual amplió el escenario de indagación. El proceder ético estuvo enmarcado en tres aspectos: primero, el contacto con los participantes directamente sin que mediaran los cuidadores, de tal manera que se mantuviera la coherencia de la perspectiva política del estudio. Segundo, se aplicó la Escala de Intensidad de Apoyos (en inglés Support Intensity Scale - sIs) (Thompson, Bryant, Campbell, Craig, Hughes, Rotholz, Silverman, Tasse y Wehmeyer, 2004), para definir que las personas entrevistadas tenían la capacidad de decidir su participación en la investigación de manera voluntaria, con los apoyos necesarios como el acompañamiento, en algunos casos, de uno de sus cuidadores. Por último, se contó con el consentimiento informado como documento legal de responsabilidad con los datos y la información que las personas participantes entregaran. Durante la firma de este documento, se explicitaron los objetivos de la investigación, el interés científico y académico de la misma y el compromiso de salvaguardar la identidad y otros datos personales.

El conocimiento aquí construido se obtuvo por medio de la aproximación a la realidad, para comprenderla, tanto en su lógica interna, como en su especificidad (Sandoval-Casilimas, 2002). Los relatos de vida permitieron reconocer a los participantes como actores activos de sus realidades (Galeano-Marín, 2004). En este argumento se halla la importancia de los métodos comprensivos, puesto que captar el sentido de las acciones humanas requiere el reconocimiento del carácter significativo de estas acciones (Durán-Palacio, 2018), más allá de la explicación causalista que ha imperado en la producción de conocimiento que sigue el método científico de las ciencias naturales. En este sentido, se otorga importancia a la experiencia hermenéutica, entendida como la situación interpretativa en la que siempre está el ser humano (Herrera, 2009), y en la cual se tiene presente la tensión entre el texto producido por los participantes, a través de su narrativa y el acto interpretativo del investigador, quien debe tener un marco de referencia epistemológico, teórico y metodológico para acceder a los sentidos de esa experiencia narrada, de la manera más fiel posible. Asimismo, se parte del argumento de que la manera de acceder al significado de las vivencias es posible a través de herramientas interactivas que favorezcan la conversación y que revelen el sentir de los participantes; por ello, la entrevista en profundidad (Valles-Martínez, 2002) fue el instrumento más acorde para el propósito del estudio, y, para organizar la información generada, se utilizaron la herramienta Atlas Ti y las matrices de análisis. El análisis se realizó con base en Coffey y Atkinson (2003) y Strauss y Corbin (2002), con el propósito de trascender el carácter descriptivo y lograr develar sentidos que favorecieran la comprensión de los relatos. Como herramienta secundaria para este proceso, se utilizó el análisis de contenido (Ruiz-Silva 2006). 


\subsection{Participantes}

La selección de los participantes fue de manera intencional y por conveniencia. En tanto la pregunta de investigación estaba encaminada a develar la relación entre vivencias de reconocimiento y compasión y desarrollo de autonomía y vida independiente, uno de los criterios es que las personas seleccionadas mantuvieran actividades sociales, laborales, escolares o familiares que permitieran revelar esta autonomía e independencia.

Se seleccionaron cuatro participantes que cumplieran con los siguientes criterios de inclusión:

- Personas con discapacidad física, visual, cognitiva y múltiple. Se tuvo en cuenta la definición de los anteriores términos desde la Clasificación Internacional del Funcionamiento, de la Discapacidad y de la Salud (CIF).

- Que informaran sobre la presencia de la discapacidad desde su niñez, ya fuera adquirida o de nacimiento.

- Con una edad cronológica entre los 30 y 40 años de edad, con el fin de identificar prácticas vivenciadas en los diferentes ámbitos de participación a lo largo del ciclo vital individual, que evidencien acciones de independencia y autonomía de los participantes.

- Representatividad de ambos géneros (femenino y masculino)

Asimismo, se tuvieron en cuenta para la caracterización por participante las variables de análisis identificadas por Iáñez-Domínguez (2009) como:

- Demográficas: edad, sexo, estado civil, hábitat, nivel de estudios, tipo de empleo, ingresos y procedencia de los mismos, unidad de convivencia.

- Discapacidad: tipo, grado, origen, momento de aparición, evolución, habilidades funcionales, servicios requeridos para la autonomía física.

- Entorno: red social, alojamiento, ayudas técnicas, accesibilidad, ocio y tiempo libre.

\begin{tabular}{|c|c|l|l|l|l|l|l|}
\hline \multicolumn{7}{|c|}{ TABLA 1. Información de los participantes } \\
\hline $\begin{array}{c}\text { Partici- } \\
\text { pante }\end{array}$ & $\begin{array}{c}\text { Edad } \\
\text { (años) }\end{array}$ & Género & $\begin{array}{c}\text { Tipo de } \\
\text { discapacidad }\end{array}$ & $\begin{array}{c}\text { Actividad } \\
\text { económica } \\
\text { principal }\end{array}$ & $\begin{array}{c}\text { Nivel de } \\
\text { formación }\end{array}$ & $\begin{array}{c}\text { Otras } \\
\text { actividades }\end{array}$ & $\begin{array}{c}\text { Personas } \\
\text { con quienes } \\
\text { vive }\end{array}$ \\
\hline PCDF & 40 & Mujer & $\begin{array}{l}\text { Física desde el } \\
\text { nacimiento }\end{array}$ & $\begin{array}{l}\text { Prestadora de } \\
\text { servicios con } \\
\text { Alcaldía de } \\
\text { Medellín }\end{array}$ & Técnica & $\begin{array}{l}\text { Vendedora por } \\
\text { catálogo. } \\
\text { Prácticas } \\
\text { deportivas }\end{array}$ & Hermana \\
\hline PCDV & 31 & Hombre & $\begin{array}{l}\text { Visual desde el } \\
\text { nacimiento }\end{array}$ & $\begin{array}{l}\text { Profesional en } \\
\text { Planeación y } \\
\text { Desarrollo Local } \\
\text { de la Universidad } \\
\text { Pascual Bravo }\end{array}$ & Universitario & Solo & \\
\hline
\end{tabular}


VIVENCIAS DE RECONOCIMIENTO Y COMPASIÓN DE PERSONAS EN CONDICIÓN DE DISCAPACIDAD. HACIA LA AUTONOMÍA Y LA VIDA INDEPENDIENTE

NATALI ROLDÁN BERRIO Y MAGDA VICTORIA DÍAZ ALZATE

\section{TABLA 1. Información de los participantes (cont.)}

\begin{tabular}{|l|c|l|l|l|l|l|l|}
\hline $\begin{array}{c}\text { Partici- } \\
\text { pante }\end{array}$ & $\begin{array}{c}\text { Edad } \\
\text { (años) }\end{array}$ & Género & $\begin{array}{c}\text { Tipo de } \\
\text { discapacidad }\end{array}$ & $\begin{array}{c}\text { Actividad } \\
\text { económica } \\
\text { principal }\end{array}$ & $\begin{array}{c}\text { Nivel de } \\
\text { formación }\end{array}$ & \multicolumn{1}{c|}{$\begin{array}{c}\text { Otras } \\
\text { actividades }\end{array}$} & $\begin{array}{c}\text { Personas } \\
\text { con quienes } \\
\text { vive }\end{array}$ \\
\hline PCDM & 38 & Mujer & $\begin{array}{l}\text { Mental y } \\
\text { física desde el } \\
\text { nacimiento }\end{array}$ & Desempleada & Bachiller & $\begin{array}{l}\text { Estudiante de } \\
\text { Lengua de señas } \\
\text { colombiana. } \\
\text { Integrante del } \\
\text { Comité Comunal } \\
\text { de Inclusión de la } \\
\text { Comuna 12. } \\
\text { Prácticas } \\
\text { deportivas }\end{array}$ & Hermana \\
\hline PCDC & 38 & Hombre & $\begin{array}{l}\text { Cognitiva con } \\
\text { origen durante } \\
\text { el parto }\end{array}$ & $\begin{array}{l}\text { Trabajador } \\
\text { independiente }\end{array}$ & $\begin{array}{l}\text { Técnico en } \\
\text { fotografía } \\
\text { ybailarín } \\
\text { profesional }\end{array}$ & $\begin{array}{l}\text { Bailarín. } \\
\text { Prácticas } \\
\text { deportivas }\end{array}$ & $\begin{array}{l}\text { Padres y } \\
\text { hermanos }\end{array}$ \\
\hline
\end{tabular}

Fuente: Elaboración propia de las autoras.

\subsection{Estrategias de recogida y análisis de los datos}

El contacto inicial con las personas participantes lo realizó una de las investigadoras, que conocía cada caso con anterioridad por su actividad laboral. Teniendo en cuenta la definición de los participantes descrita en el apartado anterior para la selección, se definieron las personas cercanas, que permitieran el acceso a su historia de vida y cumplieran con los criterios de inclusión. Para acceder a los relatos, se precisa la entrevista en profundidad o cualitativa (Valles-Martínez, 2002), que permite rescatar los sentidos construidos por los participantes, que son la manera en que las personas narran las experiencias a partir del significado y la interpretación que le hayan otorgado para sí mismas y para la interacción social (Chárriez Cordero, 2012). Se realizaron tres entrevistas por cada participante que fueron grabadas en audio y transcritas, manteniendo la fidelidad de las expresiones, lo que arrojó el texto para analizar.

Además, como se mencionó en el diseño de la investigación, se utilizó la sis (Thompson et al., 2004), que fue diseñada para: a) evaluar las necesidades de apoyo, b) determinar la intensidad de los apoyos necesarios, c) supervisar los progresos y d) evaluar los resultados en adultos con discapacidad intelectual y discapacidades del desarrollo. Para el caso del presente estudio, se tuvieron en cuenta las necesidades de apoyos y la intensidad de los mismos, en dos sentidos: uno, que tiene que ver con las consideraciones éticas, en cuanto los resultados muestran que las personas entrevistadas tienen la capacidad de decidir su participación en la investigación de manera voluntaria, con los apoyos necesarios como el acompañamiento, en algunos casos, de uno de sus cuidadores. El segundo sentido de la aplicación de la escala tuvo que ver con la construcción de la caracterización de la población, de manera ética, política y 
más aproximada a la comprensión de sus cuerpos diversos, de sus condiciones socioeconómicas, de los apoyos requeridos y de su ser político. Para la aplicación de esta escala, se utilizó la entrevista, y este momento narrativo permitió profundizar en las maneras de vivir la autonomía y la independencia.

El análisis de la información se concibió como un tejido entre los saberes y experiencias narradas por los participantes y el proceso de interpretación de las investigadoras, en el cual cobra relevancia la relación intersubjetiva, mediada por los objetivos y los marcos de referencia del estudio. Fue importante comprender que esta relación no invalidaba el proceso de construcción de conocimiento, puesto que se concibe que la relación empática es importante para acceder a las experiencias y develar los sentidos que han acuñado los participantes en estos acontecimientos. Al decir de Strauss y Corbin (2002), “Tener sensibilidad significa ser capaz de penetrar y dar significado a los acontecimientos y sucesos que muestran los datos. Significa ver más allá de lo obvio para descubrir lo nuevo” (p. 52). El análisis de la información generada se realizó con base en las propuestas de Coffey y Atkinson (2003) y de Strauss y Corbin (2002), de manera que la codificación y la categorización de los datos pudiesen trascender la descripción para la construcción de sentidos más profundos sobre las vivencias de las personas en condición de discapacidad.

En este proceso, se utilizó el análisis de contenido como herramienta metodológica secundaria (Ruiz-Silva, 2004) para organizar la información transcrita y profundizar en las expresiones más recurrentes de los participantes. En cuanto a la fiabilidad y validez, se acude al proceso de triangulación. En un primer momento, se presentaron las interpretaciones construidas a los participantes, quienes validaron lo elaborado a partir de sus relatos. Este momento se nombró como triangulación con la fuente

\begin{tabular}{|l|l|}
\hline \multicolumn{2}{|c|}{ TABLA 2. Ejemplo de análisis } \\
\hline \multicolumn{2}{|c|}{ Categoría final 3. Experiencias de reconocimiento y compasión, que favorecen } \\
la independencia y la autonomía
\end{tabular}

Fuente: Elaboración propia de las autoras.

() Ediciones Universidad de Salamanca / CC BY-NC-ND

Siglo Cero, vol. 50 (3), n. ${ }^{\circ} 271,2019$, julio-septiembre, pp. 67-87 
primaria. En un segundo momento, se realizó una contrastación (Martínez-Miguélez, 2004) documental con los antecedentes construidos y los referentes teóricos, y un intercambio con investigadores de la Línea de Investigación Problemáticas Psicosociales Contemporáneas de la Universidad Católica Luis Amigó. Este primer momento se nombró como triangulación con fuentes secundarias. A partir de estas triangulaciones, se construyeron las categorías finales, que se constituyen en los hallazgos de la investigación.

\section{Resultados}

Se presentan dos categorías emergentes como parte de los resultados de la investigación. La primera da cuenta de las vivencias de reconocimiento y compasión en los diferentes ámbitos que han servido de escenarios de socialización y participación. Aquí se rescatan los espacios y las personas acompañantes y cuidadores que han favorecido estas vivencias. La segunda categoría presenta un análisis relacional entre las vivencias de reconocimiento y compasión y el desarrollo de la autonomía y la configuración de estilos de vida independiente.

FIGURA 1. Relaciones para el desarrollo de la autonomía y la configuración de estilos de vida independiente

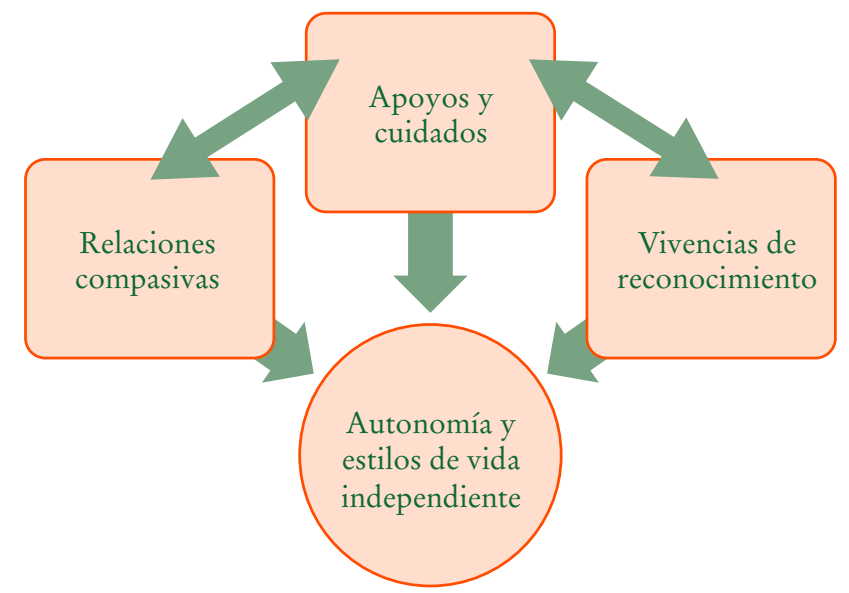

Fuente: Elaboración provia de las autoras.

\subsection{Vivencias de reconocimiento y compasión en ámbitos de socialización y participación}

Según las experiencias de los participantes, los ámbitos en los cuales vivieron experiencias de reconocimiento y compasión fueron familiar, laboral, deportivo, comunitario, hospitalario y educativo. Estos ámbitos son espacios en los cuales aparece 
el cuerpo como fenómeno social (Butler, 2010), es decir, como herramienta que permite el encuentro entre las personas. Si se habla de personas en condición de discapacidad, se tiene que nombrar que estos cuerpos son diversos, que tienen limitaciones y requieren apoyos para su desempeño cotidiano. Dichos ámbitos, más que lugares físicamente dados, se han convertido en espacios simbólicos donde se cumplen o no las expectativas de vida, a partir del proceso de configuración dado en su círculo más íntimo a cada uno de estas personas. Allí han adquirido herramientas, que les permiten alcanzar niveles afectivos importantes para la aceptación en un entorno discapacitante, para tomar una actitud positiva hacia sí mismos y para el surgimiento de relaciones vinculares con sus pares, permeadas por condiciones de solidaridad y protección.

A partir de esta participación en diferentes ámbitos, se encuentran relatos relacionados con la vivencia del reconocimiento. Al decir de Honneth (1996), "reconocer a alguien en un sentido determinado de su integridad personal sólo puede denotar aquellos actos a realizar o los criterios a adoptar para que pueda alcanzar la comprensión correspondiente de su propia persona” (p. 15), y ello es evidente en la narración de PCDM: "saludo a mis compañeros del INDER, ya no les agacho la cabeza ni a los profesores, ni a nadie, pues ni a mi familia, no. Y pues lo superé, pues normal porque mi familia me ayudó a seguir adelante" (comunicación personal, 19 de febrero de 2018).

De igual manera, la experiencia amorosa propuesta por Honneth en la primera esfera de reconocimiento se hace más contundente en las relaciones con familiares como tíos y abuelas, como lo expresa PCDV al recordar que en el proceso inicial de reconocimiento "tuvo mucho que ver un tío, que fue el primero que le hizo caso a una locura que tuve, que fue crear un programa de televisión como ya lo menciona, de ahí salió el proyecto Ser Capaz” (comunicación personal, 2 de septiembre de 2017). Aquí, no solo se favorece el desarrollo de la autoconfianza que es el fin que propone Honneth en esta esfera del amor, sino que se trasciende a la acción, a la construcción de un proyecto de vida y laboral. Para las personas en cualquier condición, el acompañamiento amoroso y la confianza de los más cercanos son imperativos para que se desarrolle esta autoconfianza. Si bien para Erikson (1998) la confianza tiene su hito en el primer momento del desarrollo psicosocial ( 0 a 12-18 meses de edad), en relatos se evidencia que no es un desarrollo exclusivo de este estadio del ciclo vital.

Con respecto a la segunda esfera que hace énfasis en los derechos o al cumplimiento de la ley a partir del reconocimiento de las personas como integrantes de una sociedad, la discapacidad ha hallado reconocimiento jurídico, lo que evidencia un tránsito hacia el enfoque de derechos, la participación y la identificación de las necesidades de apoyo, trascendiendo así la concepción de beneficencia (Zorrilla, Verdugo, Gómez Sánchez, Ezquerra y Fernández, 2015), propia de los modelos médico y social. La incesante participación de las personas en condición de discapacidad ha obligado a pasar de concepciones negativas de la discapacidad desde el modelo de la presidencia, modelos médicos que la conciben como una enfermedad, hasta el modelo más integral o de diversidad funcional (Palacios y Romañach, 2006), que reconoce sus responsabilidades y capacidades. 
Este reconocimiento genera cierto nivel de libertad que, más allá de entenderse como una situación intrínseca en el ser humano, es una condición que favorece la percepción de seguridad frente a sus capacidades, sin desconocer la existencia de ese otro y sin dejar de comprender la necesidad de interacción con este para el surgimiento del reconocimiento. Este es el sentido que se halla en el relato de PCDV cuando expresa:

Yo dejo de ser ese individuo que pensaba en sí mismo cuando empiezo a interactuar con el otro, cuando me veo en una perspectiva ecológica cierto, cuando interactuamos por el conocimiento, cuando interactuamos por las ganas, cuando sentimos que hay una necesidad pero que también hay una solución, ahí es donde yo pienso que empieza a brillar mi vida. (comunicación personal, 02 de septiembre de 2017).

La vivencia del reconocimiento jurídico, favorece las condiciones necesarias para el desarrollo profesional, personal y laboral. Trascender la perspectiva de la beneficencia hacia el reconocimiento de estas personas como ciudadanos, con necesidades de apoyo, con cuerpos diversos, con derechos a tener una vida digna, favorece la confianza en sí y con ello, el aprovechamiento de las capacidades diversas con las que cuenta. Así lo relata PCDV:

Soy una persona de 30 años [en realidad, tiene 31] que desde mis 26 tengo mi casa propia, que soy profesional universitario, que tengo un buen trabajo, que cuento con unas buenas relaciones sociales en toda la ciudad, incluso en el país y en otros países, eso para mí ya es el todo (comunicación personal, 2 de septiembre de 2017).

En cuanto a la esfera de la valoración social donde el ser humano logra fortalecer su autoestima, la participante PCDM expresa que "El INDER ${ }^{2}$ me ha enseñado mucho, el Comité $e^{3}$ e ha enseñado mucho a comunicarme con las personas, a mirarlas de frente. Por ejemplo, cuando salgo con mi familia a fiestas, me comunico pues mucho con las personas” (comunicación personal, 19 de septiembre de 2018). Esta esfera implica la interacción, puesto que solo puede darse en relación con otros, quienes otorgan un lugar dentro de los grupos, y así favorecen el desarrollo de lo que Honneth (2001, 2004) nombra cómo autoestima, y que se relaciona con el mérito, es decir, aquello que se recibe como parte de una relación de aprobación solidaria (Honneth, 1992), que además permite alcanzar las metas personales y una construcción colectiva de sociedad, donde dicha apreciación se hace fundamental para la verdadera inclusión de la población con discapacidad.

De igual modo, la valoración social se manifiesta a través de los logros obtenidos en el ámbito educativo. Los participantes ratifican la importancia de estar vinculados en espacios como la escuela o la universidad, que afianza las capacidades para lograr las metas contempladas, así lo menciona PCDF: “En ese tiempo pude lograr en dos

2 Instituto de Deportes y recreación de Medellín. Es una entidad gubernamental adscrita a la Alcaldía de la ciudad de Medellín, Colombia, en la cual se fomentan la actividad física, el deporte y la recreación de todos los ciudadanos en cualquier condición y en todas las edades.

3 Se refiere al Comité Comunal de Inclusión de la Comuna 12 de la ciudad de Medellín, en el cual se desarrollan programas gubernamentales para las personas en condición de discapacidad. 
años, pude lograr muchas cosas, porque terminé mi primaria, comencé mi bachillerato y empecé ya como otra vez a retomar mi vida, pero porque yo retomé mis riendas" (comunicación personal, 02 de septiembre de 2017).

Estas vivencias de reconocimiento, como una emoción loable en las relaciones en las cuales se resalta el enfoque de derechos y la comprensión de la pluralidad como condición humana que favorece saberse iguales y diferenciados a la vez (Arendt, 2005), se relacionan con la capacidad de sentir compasión por el otro. Estas experiencias vividas por los participantes, entendidas como la posibilidad de tener una emoción de dolor frente al padecimiento de un infortunio de otro ser (Nussbaum, 2008), implican necesariamente el reconocimiento de lo humano, de la fragilidad de sí mismo en el otro, y dota al espectador de lo que Díaz-Alzate, Arbeláez-Gómez y David-Manco (2015), apoyadas en Nussbaum, definen como "capacidad de juicio sobre un sufrimiento que [se] considera inmerecido, sea cual fuere la acción que lo suscitara” (p. 110).

Las situaciones donde los participantes vivenciaron de alguna manera la compasión por parte sus familiares, amigos o en las diferentes instituciones fueron sentidas por ellos como apoyos importantes, frente a su condición. Es el caso de PCDV, quien resalta la importancia de tener acompañamiento y apoyo, incluso económico, que le permitiera emerger en situaciones de desventaja. Define que sus necesidades primeras fueron: "tener un techo, tener una comida, tener en algún momento un apoyo emocional, independiente de cualquier vejamen o de los vejámenes que nos pone la vida" (comunicación personal, 2 de septiembre de 2017), y que las recibió de su familia. Además, reconoce en sus relaciones familiares la capacidad de acompañamiento en la condición de discapacitado. Cuando se le pregunta por las personas que ha sentido como apoyo, responde: “Tíos. Influye mucho las ganas de superarme y de ellos en que yo me superara” (PCDV, comunicación personal, 2 de septiembre de 2017). Reconoce que sus familiares no percibían culpabilidad alguna en su condición de salud, sino que tenían relaciones en igualdad de condiciones que suscitaban el cumplimiento de sus logros y la superación de los obstáculos. PCDV considera que la familia se ha relacionado con él a partir de su ser y no solo a partir de su condición, y que ello le ha permitido comprender que se necesitan apoyos en cualquier circunstancia.

Es importante diferenciar el sentimiento suscitado en la perspectiva de la beneficencia con la emoción de la compasión. En el primer caso, lo que motiva a la persona a volcarse para acompañar y apoyar al otro es la concepción de que esa persona tiene menos recursos, no solo económicos, sino emocionales y cognitivos, por su padecimiento. Es la reducción del ser humano a la condición de discapacitado. Mientras la emoción de la compasión requiere la valoración del acontecimiento sufrido por el otro a partir de la evaluación de la gravedad, del merecimiento y del reconocimiento de la condición de fragilidad propia de los seres humanos. En la relación compasiva, el otro es reconocido como igual, en tanto frágil, capaz y humano, lo que indica que no puede negar la responsabilidad para la recuperación, la elaboración de traumas o la reconstrucción de sentidos de vida. Además, implica reconocerse como frágil para disponerse a esta relación: 
El apoyo es importantísimo, es el, es el darle ánimos a la persona, el estar con la persona, el darle, el entregarle lo mejor, el que le dé ánimos, el que [sic] si le gusta algún arte o algún deporte al hombre, pues apoyarlo (PCDC, comunicación personal, 19 de febrero de 2018).

La trascendencia de la beneficencia hacia la relación compasiva admite que el sufrimiento de la otra persona y su acompañamiento por este padecimiento haga parte de la propia existencia (Nussbaum, 2008), de tal forma que el volcamiento emocional para apoyarlo no sea el interés individual, sino el bienestar de quien sufre:

Me enseñaban, me decían “vea esto. Se suma más 8, más 8, más 9, a 10 le quitamos 8 , y nos quedan y nos quedan 2" y así, me tenían paciencia pues con las multiplicaciones, con las divisiones, con las, con las sumas, con las restas (PCDM, comunicación personal, 19 de febrero de 2018).

No obstante, se hace necesario aclarar que hubo eventualidades donde los participantes no percibieron la compasión en el otro, como en el caso de la persona con discapacidad visual: "Tuve muchas dificultades como el acceso y la inclusión a la educación, porque siempre tuve el estigma de mi padre y el estereotipo de que ¿por qué era ciego?, que ¿por qué el hijo le había salido cieguito?” (PCDV, comunicación personal, 2 de septiembre de 2017), afirmando la discapacidad como una situación de sufrimiento al interior de las familias, ante una sociedad callada e indiferente.

\subsection{Experiencias de reconocimiento y compasión, que favorecen la autonomía y configuran estilos de vida independiente.}

Las experiencias de reconocimiento y compasión vividas por los participantes de la investigación se entienden como posibilidades válidas para que las personas con discapacidad logren autonomía y configuren un estilo de vida independiente acorde a sus situaciones, dependiendo del apoyo y acompañamiento que hayan recibido en sus relaciones representativas y de las circunstancias tanto negativas como positivas vividas en los diferentes ámbitos de participación donde estuvieron y continúan en interacción.

Estas experiencias que dignifican la vida favorecieron procesos de autodeterminación para la ganancia de autonomía. De ello da cuenta el relato de PCDF, al referirse al proceso de crianza con su abuela: "Me enseñó muchas cosas, eso fue lo más importante, que nunca me tuvo como la pobrecita, que no iba a ser capaz de hacer nada, que hay que hacerle todo, no" (comunicación personal, 2 de septiembre de 2017). En este testimonio, se devela la vivencia de la primera esfera del reconocimiento propuesta por Honneth, a partir de la concepción de amor de Hegel: "En el amor [...], los s ujetos [de la cita] se reconocen recíprocamente en su singular necesidad natural de tal modo que logran una seguridad afectiva en la articulación de sus exigencias instintivas" (Honneth, 1996: 8), y es precisamente esta seguridad afectiva lo que favorece el desarrollo de la confianza en sí mismo que puede ser la motivación emocional para

(C) Ediciones Universidad de Salamanca / CC BY-NC-ND

Siglo Cero, vol. 50 (3), n. ${ }^{\circ}$ 271, 2019, julio-septiembre, pp. 67-87 
la construcción de proyectos de vida que favorezcan la autonomía y el estilo de vida independiente. Así lo relata PCDV:

Soy una persona de 31 años que desde mis 26 tengo casa propia, soy profesional universitario, tengo un buen trabajo, cuento con unas buenas relaciones sociales en toda la ciudad, incluso en el país y en otros países, eso para mí ya es el todo, es la ganancia y eso me lo permitió el salir solo, el estar solo (comunicación personal, 2 de septiembre de 2017).

Para Iáñez-Domínguez (2009), la posibilidad de configurar un estilo de vida independiente está directamente relacionada con la reflexión que hacen las personas sobre sus propias experiencias subjetivas. Esta capacidad de mirarse para reconocer las potencialidades y las limitaciones puede entenderse como la consciencia que se va desarrollando en cada persona sobre su propio actuar, sobre sus fortalezas y sobre las experiencias que le han favorecido o no para su desarrollo humano. Aquí se sostiene la idea de que, si se ha desarrollado la confianza en sí mismo, en el proceso de autorreflexión se encontrarán capacidades para el logro de metas en términos de autonomía e independencia:

De los hospitales las enfermeras me ensañaron cómo cuidarme por mi salud, cómo aprender a manejar mi discapacidad, a ser muy independiente, a tener autocuidado para que no tenga más adelante alguna lesión, una discapacidad y que me quisiera como era yo, o sea, enfrentar mi mundo (PCDF, comunicación personal, 2 de septiembre de 2017).

Para los participantes, aceptar que requieren apoyos y cuidados les ha permitido reflexiones importantes sobre sus grupos de acogida y los ámbitos de participación: "El acompañamiento de la familia es muy importante, desde muy niño hasta la adolescencia porque es ahí donde la persona se forma y aprende a ser muy independiente" (PCDF, comunicación personal, 14 de febrero de 2018). La autonomía moral, como se entiende desde el concepto de vida independiente descrito por Maraña (2004), es un objetivo que no descarta los apoyos, más bien, reviste de importancia las relaciones de cuidado y acompañamiento, puesto que no debe ser entendida como autosuficiencia. En el logro de la autonomía intervienen factores como la presencia de cuidadores responsables, familiares que fortalecen la confianza para la realización de las actividades de la vida cotidiana: "Yo me superé con mi familia, porque cuando vivía con mi papá, siempre me llevaba a médicos y a psiquiatras” (PCDM, comunicación personal, 22 de septiembre de 2017).

Si bien gran parte de los relatos rescatan el ámbito familiar como importante para el desarrollo de la confianza en sí, y como aquel que les ha permitido superar la dependencia e iniciar el proceso de individuación (Fernández y Vasco, 2012), es importante presentar otras experiencias como la de PCDV, cuando se refiere a aquello que le permitió la configuración de un estilo de vida independiente: "Los aprendizajes de vida, la calle, porque a pesar de haber tenido una familia, no tuve un proceso de crianza normal" (comunicación personal, 2 de septiembre de 2017). Estos contrastes en los relatos permiten decir que si bien se ha mantenido la idea de la familia como el ámbito de cuidado y apoyo para la superación de las condiciones adversas, también 
existen otros ámbitos de acogida, condiciones personales y capacidades individuales que interactúan como factores favorecedores del desarrollo de autonomías y de configuraciones de vida independiente en los participantes de la investigación: "Hago deporte en el INDER, en el Estadio, me gusta mucho el deporte, me gusta caminar mucho, estudio lengua de señas en ASANSO"4 (PCDM, comunicación personal, 22 de septiembre de 2017).

Aquí, el enfoque de derechos sobre la discapacidad se hace plausible toda vez que se entiende el desarrollo de la autonomía en la interrelación del cuidado y el apoyo y la acción misma de las personas (CEPAL, 2012), es decir, es la trascendencia del modelo médico y del modelo social, en tanto ya no es solo la persona en relación con su condición o las barreras de la sociedad las que definen el desarrollo de esta autonomía. Ahora es una urdimbre entre la capacidad de agencia de la persona con discapacidad y las relaciones de reconocimiento y compasión de quienes cuidan y apoyan, que favorece el desarrollo de la autonomía y la configuración de estilos de vida independientes. Al respecto, PCDF dice: "Cuando hemos dado con casas que no tienen ninguna barrera, yo no tengo ninguna necesidad, ningún apoyo, porque yo puedo entrar y salir sola y en el barrio yo me movilizo sola" (comunicación personal, 14 de febrero de 2018).

La valoración social (Honneth, 2001, 2004) y la capacidad de relacionamiento solidario propio de la tercera esfera del reconocimiento propuesta por Honneth (1992) tienen correspondencia con la necesidad de configurar un estilo de vida independiente. Estos participantes saben que los apoyos externos son indispensables para el desarrollo de su autonomía, como lo menciona PCDC: "Para que las personas con discapacidad se recuperen, sigan adelante se superen, se independicen o tengan derecho a estudiar, una carrera" (comunicación personal, 19 de febrero de 2018). El reconocimiento de sus capacidades, les permite hallarse como personas activas, que participan en las decisiones sobre su propio desarrollo (Iáñez-Domínguez, 2009: 11), de acuerdo a la dimensión política asociada a la participación en sociedad y a la dimensión de lo personal relacionada con la valoración de los logros particulares.

El reconocimiento desde la valoración social, y las acciones que devienen de la compasión permiten construir condiciones necesarias para que las personas en condición de discapacidad alcancen autonomía y configuren un estilo de vida independiente, sin desconocer las limitaciones de sus cuerpos, puesto que la vida independiente necesariamente no estipula la ausencia de apoyos para la realización de actividades cotidianas, y más bien se refiere a la capacidad para tener control pleno sobre la propia vida (Iáñez-Domínguez, 2009). La compasión como la emoción que permite a los seres humanos saberse iguales en tanto frágiles y con las mismas probabilidades de sufrimiento permite reconocer que los apoyos son necesarios para la mitigación de las limitaciones en cualquier ser humano, pero que también hay circunstancias en las cuales no se requiere acompañamiento. De allí que la capacidad de controlar plenamente la vida, que es la evidencia de que se ha configurado un estilo de vida independiente, no impida solicitar ayuda en las circunstancias necesarias, sin que ello implique pérdida de autonomía para la toma de decisiones: "Importantísimo es el darle ánimos a la persona, el estar con la persona, el entregarle lo mejor, el que le dé ánimos, el que, si

4 Asociación Antioqueña de Personas Sordas. 
le gusta algún arte o algún deporte al hombre, pues apoyarlo” (PCDC, comunicación personal, 19 de febrero de 2018).

Básicamente es en movimientos que yo no capto por la discapacidad que tengo [se refiere a cuándo necesita ayuda], sí, por el tema de no ver. Es que me indiquen qué movimiento están haciendo, pero por ejemplo para caminar, para salir con mi perra, con el bastón, no (PCDV, comunicación personal, 21 de febrero de 2018).

El juicio de inmerecimiento contenido en la capacidad compasiva, al considerar que las situaciones inicialmente negativas son ajenas quien las padece, favorece que las personas en condición de discapacidad comprendan la importancia de los apoyos para su propio desarrollo y la configuración de una vida independiente:

Esto ayuda hacer un análisis en cuanto a quién soy yo, a qué cosas puede hacer una persona con x tipo de discapacidad, en mi caso por ejemplo una discapacidad visual y cómo desenvolverme en el medio, de quién a veces es la dificultad cuando una persona tiene una discapacidad, porqué el entorno nos pone barreras, porqué nosotros mismos como personas nos ponemos barreras (PCDV, comunicación personal, 21 de febrero de 2018).

Cuando Nussbaum (2008) hace referencia a la compasión como aquella emoción que anima al pensamiento a centrarse en el sufrimiento del otro y en promover acciones necesarias para que su vida sea buena en la medida de las posibilidades, hay un llamado para que el cuidado de las personas en condición de discapacidad, además de contener hechos que generen apoyos, evidencien un acompañamiento de manera justa y necesaria en la superación de la condición desventajosa.

\section{Conclusiones}

Para favorecer las condiciones de autonomía y la configuración de estilos de vida independiente, deben desarrollarse procesos de autodeterminación y de libertad para la toma de decisiones (CEPAL, 2012), lo cual habla de la importancia de reconocer a las personas en estas condiciones como agentes en su propio desarrollo, y ello trasciende la mirada de beneficencia y ubica las relaciones con ellos a partir de su reconocimiento como valioso por el solo hecho de ser humano, y de la capacidad de sentir compasión por sus padecimientos, de tal forma que se visibilice su condición de fragilidad, como parte del significado de la especie humana.

Estos desarrollos son posibles cuando las personas en condición de discapacidad se perciben agentes en los espacios de interacción cotidiana, es decir, cuando viven estos espacios como ámbitos de participación y socialización, con los apoyos necesarios para su actuación y en los cuales sientan que son cuidados, reconocidos y acompañados para el logro de sus proyectos de vida. Las personas entrevistadas viven estas experiencias con cuidadores y acompañantes, como posibilitadores de su propio florecimiento para una vida digna, con autonomía e independencia necesarias como personas de derechos. 
La capacidad autónoma y la vida independiente de estas personas no difiere de la posibilidad de tener apoyos para su funcionamiento cotidiano y el buen vivir, puesto que no se trata de desconocer las limitaciones que se tienen, sino, por el contrario, de reconocer las diferencias corporales y la diversidad como condición de la humanidad. La capacidad de entender los apoyos como necesarios para el logro de la autonomía y la independencia favorece que se perciban las vivencias de reconocimiento y compasión como necesarias para alcanzar los logros propuestos y dignificar la existencia en estas personas.

Es importante cerrar este artículo reconociendo que durante el rastreo de antecedentes investigativos se evidenció la necesidad de continuar con el desarrollo de estudios, puesto que no se hallaron investigaciones que abordaran de manera articulada la compasión, el reconocimiento, la autonomía y el estilo de vida independiente de personas en condición de discapacidad. La mayoría de los textos encontrados eran de corte teórico donde se analizaban los cambios conceptuales y normativos del concepto de discapacidad y pocos se centraban en la narrativa o la percepción de vida de las personas en condición de discapacidad, lo cual fue analizado como problemático en tanto son ellas quienes pueden dar cuenta de sus experiencias, los significados de las vivencias, el sentir frente a su condición, la autopercepción del cuerpo limitado, las necesidades de apoyo y el autorreconocimiento de las capacidades de agenciamiento.

\section{Referencias bibliográficas}

Aв.Latiff, M., Wan, W. y Azrani, M. (2015). Implementation of inclusive education for special needs learners with learning disabilities. Procedia - Social and Behavioral Sciences, 204, 81-87. doi: 10.1016/j.sbspro.2015.08.115.

Acosta Valencia, A. y Montoya Garcés, Á. (2010). Ámbitos y escenarios de participación política juvenil en Medellín. Revista Anagramas, 8 (16), 15-31. Recuperado de https://revistas.udem.edu.co/index.php/anagramas/article/view/452/407.

Allan, V., Smith, B., Côté, J., Martin Ginis, K. y Latimer-Cheung, A. (2017). Narratives of participation among individuals with physical disabilities: A life-course analysis of athletes' experiences and development in parasport. Psychology of Sport and Exercise, 29 (1), 40-50. doi: 10.1016/j.psychsport.2017.10.004.

Arduini, G. y Chiusaroli, D. (2013). Special educational needs and evaluation. Procedia - Social and Behavioral Sciences, 106, 2438-2443. doi: 10.1016/j.sbspro.2013.12.280.

Arendt, H. (2005). La condición humana. Barcelona: Paidós Ibérica S.A.

Baña Castro, M., Losada Puente, L. y Muñoz Cantero, J. M. (2015). Condicionantes personales y ambientales de la conducta autodeterminada en la adolescencia con alteraciones del desarrollo intelectual. Ciencias Psicológicas, 9 (2), 283-292. Recuperado de https:// www.redalyc.org/articulo.oa?id=459545411006.

Bossaert, G., Colpin, H., Pijl, S. J. y Petry, K. (2013). Social participation of students with special educational needs in mainstream seventh grade. Procedia - Social and Behavioral Sciences, 93, 1952-1956. doi: 10.1016/j.sbspro.2013.10.147.

Butler, J. (2002). Cuerpos que importan: sobre los límites materiales y discursivos del "sexo". Buenos Aires: Paidós. 
VIVENCIAS DE RECONOCIMIENTO Y COMPASIÓN DE PERSONAS EN CONDICIÓN DE DISCAPACIDAD. HACIA LA AUTONOMÍA Y LA VIDA INDEPENDIENTE

NATALI ROLDÁN BERRIO Y MAGDA VICTORIA DÍAZ ALZATE

Castillo, C. y Garro-Gil, N. (2015). Inclusion and integration on special education. Procedia - Social and Behavioral Sciences, 191, 1323-1327. doi: 10.1016/j.sbspro.2015.04.488.

Chárriez Cordero, M. (2012). Historias de vida: Una metodología de investigación cualitativa. Revista Griot, 5 (1), 50-67.

Coffey, A. y Atrinson, P. (2003). Encontrar el sentido a los datos cualitativos. Estrategias complementarias de investigación. Medellín: Editorial Universidad de Antioquia.

Comisión Económica para América Latina y el CARIBe - CEPAl (2012). Panorama social de América Latina: el cuidado de las personas con discapacidad en América Latina y el Caribe: una aproximación comprehensiva. Santiago de Chile: Organización de las Naciones Unidas-ONU.

Congreso de la República de Colombia (2006). Ley 1090. Bogotá: Ministerio de la Seguridad Social.

Cramerotti, S. e Ianes, D. (2016). An ontology-based system for building Individualized Education. Plans for students with Special Educational Needs. Procedia-Social and Behavioral Sciences, 217, 192-200. doi: 10.1016/j.sbspro.2016.02.062.

Departamento Administrativo Nacional de Estadística-dane (2005). Censo General 2005. Gobierno de Colombia.

Dias, P. (2015). For a qualitative leap in inclusion: illusions and delusions from teacher's perspective. Procedia - Social and Behavioral Sciences, 191, 1119-1123. doi: 10.1016/j.sbspro.2015.04.453.

Díaz-Alzate, M. V., Arbeláez-Gómez, L. M. y David-Manco, O. T. (2015). La familia como espacio de socialización política: prácticas de crianza que forman a niños y niñas en cuidado, reconocimiento y compasión (Tesis de Maestría). Universidad de Manizales y cinDE, Manizales.

Dollar, C. H., Fredrick, L., Alberto, P. y Lucke, J. (2011). Using simultaneous prompting to teach independent living and leisure skills to adults with severe intellectual disabilities. Research in Developmental Disabilities, 33 (1), 189-195. doi: 10.1016/j.ridd.2011.09.001.

Durán-Palacio, N. M. (2018). Configuración de los objetos de investigación. Fundamentos epistemológicos. En P. A. Montoya-Zuluaga y S. N. Cogollo-Ospina (Comps.), Situaciones y retos en Latinoamérica (pp. 28-43). Medellín: Fondo Editorial Universidad Católica Luis Amigó.

Erikson, E. (1998). Ciclo de vida completo. Porto Alegre: Artmed.

FERnÁNDEZ, A. y VAsco, E. (2012). Dinámicas del reconocimiento en las narraciones de jóvenes con discapacidades. Revista Latinoamericana de Ciencias Sociales, Niñez y Juventud, 10 (1), 467-479. Recuperado de http://www.scielo.org.co/pdf/rlcs/v10n1/v10n1a30.pdf.

Galeano-Marín, M. E. (2004). Estrategias de investigación social cualitativa. El giro en la mirada. Medellín: La Carreta Editores.

Gonçalves, T. y Lemos, M. (2014). Personal And Social Factors Influencing Students' Attitudes Towards Peers With Special Needs. Procedia - Social and Behavioral Sciences, 112, 949-955. doi: 10.1016/j.sbspro.2014.01.1253.

Herrera, J. D. (2009). La comprensión de lo social. Horizonte hermenéutico de las ciencias sociales. Manizales: CINDE.

Honneth, A. (1992). Integridad y desprecio. Motivos básicos de una concepción de la moral desde la teoría del reconocimiento. Isegoría, 5, 78-92. doi: 10.3989/isegoria.1992.i5.339.

Honneth, A. (1996). Reconocimiento y obligaciones morales. Revista Internacional de Filosofía Política RIFP, (8), 5-17. Recuperado de http://e-spacio.uned.es/fez/eserv. php?pid=bibliuned:filopoli-1996-8-6443431F-2BE8-F544-3A97-47F0DA074DF8\&dsID =reconocimiento_obligaciones.pdf.

(C) Ediciones Universidad de Salamanca / CC BY-NC-ND

Siglo Cero, vol. 50 (3), n. ${ }^{\circ}$ 271, 2019, julio-septiembre, pp. 67-87 
Honneth, A. (2001). Recognition or redistribution? Changing perspectives on the moral order of society. Theory, Culture and Society - Sage, 18 (2-3), 43-55.

Honneth, A. (2004). Recognition and justice. Outline of a plural theory of justice. Acta Sociologica - Sage, 47 (4), 351-364.

Honneth, A. (2006). El reconocimiento como ideología. Isegoría, 35, 129-150. doi: 10.3989/ isegoria.2006.i35.33.

Honneth, A. (2009). Reconocimiento y menosprecio: Sobre la fundamentación normativa de una teoría social. Estudios de Filosofía Práctica e Historia de las Ideas, 15 (1), 9-44.

Iáñez-Domínguez, A. (2009). Prisioneros del cuerpo. La construcción social de la diversidad funcional. Santiago de Compostela: Diversitas Ediciones.

Löve, L., Traustadóttir, R. y Rice, J. G. (2017). Trading autonomy for services: Perceptions ofusers and providers of services for disabled people in Iceland. ALTER, European Journal of Disability Research, 12 (4), 193-207. doi: 10.1016/j.alter.2018.04.008.

Maraña, J. (2004). Vida independiente. Nuevos modelos organizativos. Madrid: Asociación Iniciativas y Estudios Sociales.

Martínez Miguélez, M. (2004). Ciencia y arte en la investigación cualitativa. México: Trillas.

Mattevi, B. S., Bredemeier, J., Fam, C. y Fleck, M. P. (2012). Quality of care, quality of life, and attitudes toward disabilities: perspectives from a qualitative focus group study in Porto Alegre, Brazil. Revista Panamericana de Salud Pública, 31 (3), 188-196. Recuperado de https://scielosp.org/pdf/rpsp/2012.v31n3/188-196/en.

Ministerio de Salud y Protección Social (2018). Registro para la localización y caracterización de personas con discapacidad - RLCPD. Gobierno de Colombia. Recuperado de https://www.minsalud.gov.co/proteccionsocial/promocion-social/Discapacidad/Paginas/ registro-localizacion.aspx.

Nussbaum, M. C. (2008). Paisajes del pensamiento. La inteligencia de las emociones. Barcelona: Paidós Ibérica.

Nussbaum, M. C. (2012). Las fronteras de la justicia. Consideraciones sobre la exclusión. Barcelona: Paidós Ibérica.

Organización Mundial de la Salud-oms (2001). Clasificación Internacional del Funcionamiento, de la Discapacidad y de la Salud (CIF). Recuperado de http://www.imserso.es/ InterPresent2/ groups/imserso/documents/binario/435cif.pdf.

Organización Mundial de la Salud-oms. (2011). Informe mundial sobre la discapacidad. Malta: Banco Mundial.

Palacios, A. y Romañach, J. (2006). El modelo de la diversidad. Valencia: Diversitas Ediciones.

Pascual-García, D., Garrido-Fernández, M. y Antequera-Jurado, R. (2014). Autodeterminación y calidad de vida: un programa para la mejora de personas adultas con discapacidad intelectual. Psicología Educativa, 20 (1), 33-38. doi: 10.1016/j.pse.2014.05.004.

Peña-Ochoa, M. y Durán Palacio, N. M. (2016). Justicia organizacional, desempeño laboral y discapacidad. Revista Colombiana de Ciencias Sociales, 7 (1), 201-222. doi: 10.21501/22161201.1540.

Pérez Bustos, R., Moya Solar, E. y Jiménez Figueroa, A. (2013). Autodeterminación, apoyo familiar y expectativas de empleabilidad en estudiantes universitarios con discapacidad. Revista Internacional PEI, 3 (5), 20-45.

Rachmawati, M., Nu'man, T., Widiasmara, N. y Wibisono, S. (2016). Differentiated instruction for special needs in inclusive schools: A preliminary study. Procedia - Social and Behavioral Sciences, 217, 585-593. doi: 10.1016/j.sbspro.2016.02.053. 
Rojas, S. (2006). Autodeterminación y personas adultas con discapacidad intelectual. Revista Médica Internacional sobre el Sindrome de Down, 10 (3), 45-48. doi: 10.1016/S11382074(06)70051-3.

Rojas, S. y Haya, I. (2017). “Nos gustaría que nos acompañasen en nuestras decisiones”. Algunas cuestiones que preocupan a personas adultas con discapacidad intelectual. Siglo Cero, 48 (3), 55-72. doi: 10.14201/scero20174835572.

Ruiz-Silva, A. (2004). Texto, testimonio y metatexto. El análisis de contenido en la investigación en educación. En A. Jiménez y A. Torres, La práctica investigativa en Ciencias Sociales (pp. 45-61). Bogotá: Editorial Universidad Pedagógica Nacional.

Sandoval-Casilimas, C. (2002). Investigación cualitativa. Bogotá: Instituto Colombiano para el Fomento de la Educación Superior-ICFES.

Santamaría Pinzón, O. L., Angarita Fonseca, A., Bayona Gualdrón, L. K., Delgado Silva, J., Parra Guerrero, I. Y. y Pérez Ortega, Á. P. (2013). Asociación entre participación social de los niños con discapacidad y la satisfacción de los padres. Revista Cubana de Pediatría, 85 (1), 17-27.

Strauss, A. y Corbin, J. (2002). Bases de la investigación cualitativa. Técnicas y procedimientos para desarrollar teoría fundamentada. Medellín: Editorial Universidad de Antioquia.

Thompson, J., Bryant, B., Campbell, E., Craig, E., Hughes, C., Rotholz, D., Silverman, W., Tasse, M. y Wehmeyer, M. (2004). Supports Intensity Scale. Washington: American Association on Intellectual and Developmental Disabilities.

Valles, M. (2002). Entrevistas Cualitativas. Cuadernos Metodológicos, 32 (pp. 1-195). Madrid: Centro de Investigaciones Sociológicas.

Zorrilla, I. H., Verdugo, M. Á., Gómez Sánchez, L. E., Ezquerra, S. F. y Fernández, P. C. (2015). Evaluación de la calidad de vida en personas con discapacidades significativas: aplicación de la Escala San Martín en la Fundación Obra San Martín. Revista Española de Discapacidad, 3 (1), 93-105. doi:10.5569/2340-5104.03.01.05. 


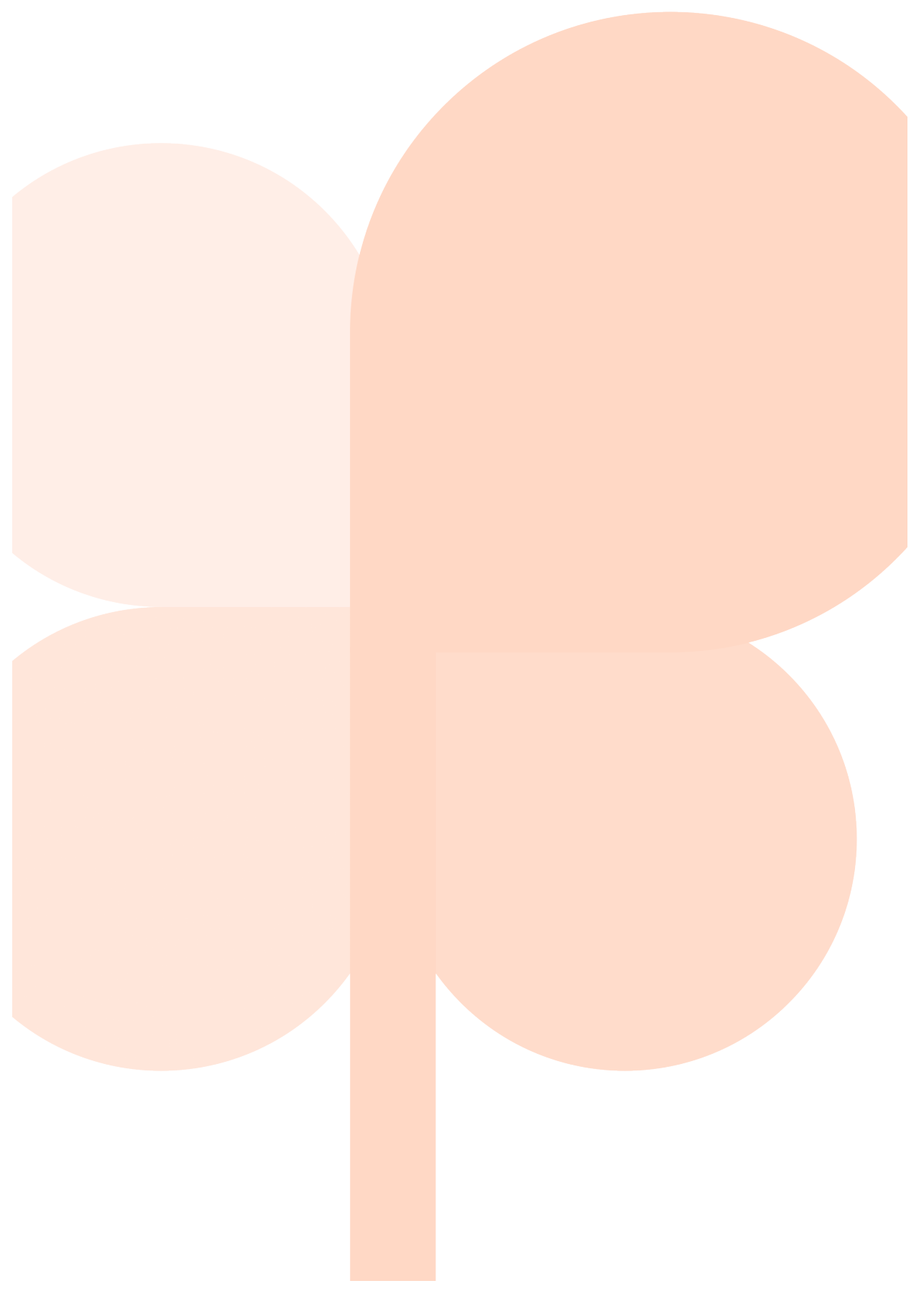

